\title{
Arsenic Methylation Dynamics in a Rice Paddy Soil Anaerobic Enrichment Culture
}

\author{
Matthew C. Reid, ${ }^{*}, \|_{\odot}$ Julien Maillard, ${ }^{\ddagger}$ Alexandre Bagnoud, ${ }^{\dagger, \perp}$ Leia Falquet, ${ }^{\dagger}$ Phu Le Vo, ${ }^{\S}$ \\ and Rizlan Bernier-Latmani ${ }^{\dagger}$ \\ ${ }^{\dagger}$ Environmental Microbiology Laboratory, École Polytechnique Fédérale de Lausanne, Lausanne CH-1015, Switzerland \\ ${ }^{\ddagger}$ Laboratory for Environmental Biotechnology, École Polytechnique Fédérale de Lausanne, Lausanne CH-1015, Switzerland \\ ${ }^{\S}$ Ho Chi Minh City University of Technology - VNU HCM, Ho Chi Minh City, Vietnam
}

\section{Supporting Information}

ABSTRACT: Methylated arsenic (As) species represent a significant fraction of the As accumulating in rice grains, and there are geographic patterns in the abundance of methylated arsenic in rice that are not understood. The microorganisms driving As biomethylation in paddy environments, and thus the soil conditions conducive to the accumulation of methylated arsenic, are unknown. We tested the hypothesis that sulfate-reducing bacteria (SRB) are key drivers of arsenic methylation in metabolically versatile mixed anaerobic enrichments from a Mekong Delta paddy soil. We used molybdate and monofluorophosphate as inhibitors of sulfate reduction to evaluate the contribution of SRB to arsenic biomethylation, and developed degenerate primers for the amplification of

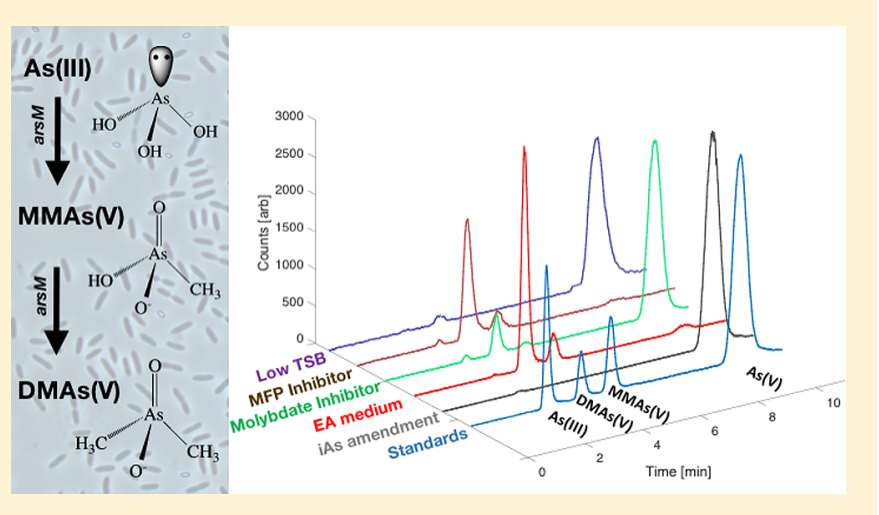
arsM genes to identify methylating organisms. Enrichment cultures converted $63 \%$ of arsenite into methylated products, with dimethylarsinic acid as the major product. While molybdate inhibited As biomethylation, this effect was unrelated to its inhibition of sulfate reduction and instead inhibited the methylation pathway. Based on ars $M$ sequences and the physiological response of cultures to media conditions, we propose that amino acid fermenting organisms are potential drivers of As methylation in the enrichments. The lack of a demethylating capacity may have contributed to the robust methylation efficiencies in this mixed culture.

\section{INTRODUCTION}

Growing awareness of the global problem of arsenic (As) contamination of rice ${ }^{1-3}$ has directed substantial research into the biogeochemical cycling and fate of As in rice paddy soils. ${ }^{4-7}$ One research priority has been to understand the environmental controls on As methylation in soils, ${ }^{8-10}$ since methylated As species are a significant fraction of As in rice grains. ${ }^{2}$ Methylated arsenic in rice originates in paddy soil, and is produced via microbial methylation of inorganic arsenic ${ }^{11}$ or is present due to legacy application of organoarsenic-based pesticides. ${ }^{12}$ Distinct geographic patterns exist in methylated arsenic abundance in rice grains, with rice grown in the southcentral U.S. tending to have a large fraction of methylated arsenic, while Asian-grown rice accumulates primarily inorganic As (iAs). ${ }^{2,8,13}$ The reasons for these patterns are unknown.

Microbial arsenic methylation is catalyzed by arsenite $S$ adenosylmethionine methyltransferase (ArsM) enzymes ${ }^{14}$ which mediate the sequential methylation of As(III) to mono-, di-, and trimethylated species. The biological function of biomethylation is controversial. ${ }^{15}$ Methylation has traditionally been understood as a detoxification process, particularly in aerobic systems in which As(III) is converted into less toxic pentavalent methylated species, or into volatile arsines which are removed from environmental media via volatilization. ${ }^{12,14,16,17}$ The formation of very toxic trivalent methylated species complicates this interpretation, however, particularly in anaerobic environments in which trivalent methylarsenicals are not oxidized to their pentavalent derivatives. ${ }^{15}$

ars $M$ genes are present in phylogenetically and functionally diverse microorganisms ${ }^{8,9,11,18-20}$ and are widely distributed in soil, freshwater, and marine ecosystems. ${ }^{21,22}$ Pure culture studies of As methylation have shown that the extent of methylation varies widely. Methanosarcina acetivorans $\mathrm{C}_{2} \mathrm{~A}^{23}$ and Clostridium sp. $\mathrm{BXM}^{24}$ transformed approximately $10 \%$ of aqueous iAs to methylated species, while Streptomyces sp., ${ }^{25}$ Pseudomonas alcaligenes ${ }^{26}$ and Arsenicibacter rosenii ${ }^{27}$ were able to transform more than half of aqueous iAs into methylated species. Aerobic organisms routinely methylate more than twothirds of aqueous iAs, while the anaerobes likely to dominate

Received: June 9, 2017

Revised: August 2, 2017

Accepted: August 21, 2017

Published: August 21, 2017 
Table 1. Summary of Arsenic Methylation Efficiencies by Prokaryotes in Pure and Mixed Culture

\begin{tabular}{|c|c|c|c|c|}
\hline strain or culture type & $\begin{array}{c}\text { maximum observed methylation } \\
\text { efficiency }\end{array}$ & major product(s) & notes & reference \\
\hline \multicolumn{5}{|c|}{ Anaerobes } \\
\hline $\begin{array}{l}\text { Enrichment Culture from Lake } \\
\text { Sediment }\end{array}$ & $12 \%$ & $\mathrm{DMAs}(\mathrm{V})$ & $\begin{array}{l}35 \mathrm{~d} \text { incubation; } 1 / 4 \text { strength TSB } \\
\text { media }\end{array}$ & Bright et al. $^{30}$ \\
\hline Clostridium sp. BXM & $10 \%$ & $\operatorname{MMAs}(\mathrm{V})$ & $20 \mathrm{~d}$ incubation & Wang et $\mathrm{al}^{24}$ \\
\hline Methanosarcina acetivorans C2A & $13 \%$ & $\operatorname{MMAs}(\mathrm{V})$ & $20 \mathrm{~d}$ incubation & Wang et al. ${ }^{23}$ \\
\hline Mixed culture enriched from paddy soil & $63 \%$ & $\operatorname{DMAs}(\mathrm{V})$ & $4 \mathrm{~d}$ incubation & This Study \\
\hline \multicolumn{5}{|c|}{ Aerobes } \\
\hline Pseudomonas alcaligenes & $80 \%$ & $\operatorname{DMAs}(\mathrm{V})$ & $4 \mathrm{~d}$ incubation & Zhang et al. ${ }^{26}$ \\
\hline Streptomyces sp. & $\sim 67 \%$ & $\begin{array}{l}\operatorname{MMAs}(\mathrm{V}) \\
\operatorname{DMAs}(\mathrm{V})\end{array}$ & $12 \mathrm{~d}$ incubation & Kuramata et al. ${ }^{25}$ \\
\hline Arsenicibacter rosenii & $99 \%$ & TMAs(III), & $24 \mathrm{~h}$ incubation & Huang et al. ${ }^{27}$ \\
\hline $\begin{array}{l}\text { Pseudomonas putida (with R. palustris } \\
\text { ars M) }\end{array}$ & $97 \%$ & $\operatorname{DMAs}(\mathrm{V})$ & $16 \mathrm{~h}$ incubation & Chen et al. ${ }^{50}$ \\
\hline
\end{tabular}

paddy soil environments typically methylate $<15 \%$ of aqueous iAs (Table 1).

Functional characterization of arsenic methylation by mixed microbial communities is lacking, however, and it is not known which, if any, members of the complex paddy soil microbial community are the principal drivers of As biomethylation. ${ }^{10}$ Organic matter amendments to paddy soil enhance As biomethylation and biovolatilization ${ }^{28,29}$ but the mechanism for this biostimulation has not been explored. Tentative links have also been drawn between sulfate-reducing bacteria (SRB) and arsenic biomethylation. Bright et al. ${ }^{30}$ showed that sulfatereducing as well as fermentative and/or methanogenic cultures enriched from lake sediments produced more methylated arsenic than iron- or manganese-reducing cultures. Mikutta and Rothwell $^{31}$ showed that $82 \%$ of the As in a sulfate- and Asimpacted peat bog was present as organoarsenic species, and Zeng et al. ${ }^{32}$ showed that the abundance of methylated As in the roots of a ginseng plant increased $263 \%$ following soil amendment with sulfate $\left(\mathrm{SO}_{4}{ }^{2-}\right)$. These studies collectively suggest that sulfur and/or sulfate reduction stimulates the biotransformation of As into methylated products, and this hypothesis is supported by functional gene array studies documenting abundant ars $M$ sequences from SRB in soils from Asia, the U.K., and the U.S. ${ }^{8,11}$ If true, this active role for SRB in arsenic methylation would parallel the well-documented role of SRB as key methylators of mercury in aquatic environments. ${ }^{33-35}$ Links between SRB and arsenic methylation have not, to our knowledge, been rigorously tested.

The goal of this study was to explore the role of SRB in arsenic methylation in paddy soils through laboratory anaerobic enrichment experiments, using paddy soil from the Mekong Delta as the microbial inoculum. The effect of SRB on arsenic methylation was assessed using the specific inhibitors of dissimilatory $\mathrm{SO}_{4}{ }^{2-}$ reduction molybdate $\left(\mathrm{MoO}_{4}{ }^{2-}\right)$ and monofluorophosphate ${ }^{36}$ to block $\mathrm{SO}_{4}{ }^{2-}$ reduction and then evaluate methylation efficiencies in the presence and absence of $\mathrm{SO}_{4}{ }^{2-}$ reduction. We also examine the diversity of microorganisms harboring ars $M$ in the parent paddy soil and in enrichment culture through ars $M$ amplification with a novel primer pair and amplicon sequencing, and explore relationships between ars $M$ diversity and arsenic-methylating activity.

\section{MATERIALS AND METHODS}

Soil Sampling, Characterization, and DNA Extraction. Soil was collected from the upper $10 \mathrm{~cm}$ of a rice paddy $\left(10.905^{\circ} \mathrm{N}, 105.079^{\circ} \mathrm{E}\right)$ in An Phu District, An Giang
Province, Vietnam, in May 2014. Samples were placed in sterile plastic bags and then immediately placed in Mylar bags which were flushed with argon before being heat sealed. Samples were double-sealed in a second Mylar bag and placed on ice for transport to Switzerland. Soil pH was determined in a $1: 1$ solution of dry soil and deionized water. Major and minor element concentrations in the soil were determined via $\mathrm{X}$-ray fluorescence (PANalytical Axios mAX). Soil chemical characteristics are summarized in Table S1 in Supporting Information (SI). Genomic DNA was extracted from soils using a MoBio Power Soil DNA Isolation Kit (MoBio, Carlsbad, CA) following manufacturer's instructions, modified to include a preliminary heating step at $70{ }^{\circ} \mathrm{C}$ for $10 \mathrm{~min}$ with periodic vortexing.

Anaerobic Enrichment Cultures. Arsenic biomethylation was assayed in enrichment cultures instead of soil slurries in order to avoid interactions between methylated As and soil minerals which could confound characterization of methylated As production and overall As balances. An enrichment medium containing a variety of electron acceptors, hereafter referred to as EA medium, was used to develop anaerobic enrichments from the paddy soil. The EA medium consisted of $6 \mathrm{mM}$ $\mathrm{NaNO}_{3}, 6 \mathrm{mM} \mathrm{Na} \mathrm{SO}_{4}, 5 \mathrm{mM}$ ferric citrate, $0.01 \%$ yeast extract, $35 \mathrm{mM}$ cellobiose, and 1/4 strength TSB (tryptic soy broth). TSB is rich in amino acids and has been used previously to enrich arsenic-methylating microbes from a lake sediment. ${ }^{30}$ EA medium was used to simultaneously enrich nitrate-, iron-, and sulfate-reducers, as well as microbes with fermentative and methanogenic metabolisms. In the initial soil enrichment, $5 \mathrm{~g}$ of wet rice paddy soil were incubated in $10 \mathrm{~mL}$ of media. After the onset of methanogenic conditions, which was determined by monitoring the headspace for methane by flame ionization gas chromatography, $1 \mathrm{~mL}$ of the culture was transferred into 10 $\mathrm{mL}$ of new, sterile growth media. This procedure was then repeated three times, after which the culture was considered to be soil-free and was stored in glycerol at $-80{ }^{\circ} \mathrm{C}$. Biogenic minerals, likely FeS (SI Figure S1), were present in the final enrichment. All manipulations were performed inside an anaerobic chamber (Coy Laboratory Products) with autoclaved materials.

Arsenic methylation experiments were conducted by amending the enrichment culture with $15 \mu \mathrm{M}$ arsenite (As(III)). The ability of the cultures to demethylate and/or further methylate monomethylarsonic acid (MMAs(V)) or dimethylarsinic acid (DMAs(V)) was tested by amending cultures with $15 \mu \mathrm{M}$ MMAs(V) or DMAs(V). As(III) was 
added as sodium arsenite (Fisher Scientific, Pittsburgh, PA, USA), DMAs(V) as sodium dimethylarsinate (ABCR, Karlsruhe, Germany), and MMAs(V) as monomethylarsonic acid (Chemservice, West Chester, PA). Five mM or $20 \mathrm{mM}$ sodium molybdate ${ }^{37}$ or $10 \mathrm{mM}$ sodium monofluorophosphate $^{36}$ were added to a subset of the experiments as specific inhibitors of dissimilatory $\mathrm{SO}_{4}{ }^{2-}$ reduction. Effects of $\mathrm{SO}_{4}{ }^{2-}$ on As biomethylation were further evaluated by varying the $\mathrm{SO}_{4}{ }^{2-}$ concentration in the initial medium from 0.8 to $6.1 \mathrm{mM}$. Effects of carbon and/or amino acid nutrition on methylation efficiency were examined by reducing the TSB supplement to media EA from $1 /{ }_{4}$ strength to either $1 /{ }_{8}$ or $1 / 40$ strength TSB. Cellobiose concentration was not changed. With each experimental condition, a parallel experiment with the standard EA media was performed and inoculated with the same glycerol stock to account for potential differences in methylation efficiency between different glycerol stocks. All media were adjusted to $\mathrm{pH} 7$ before inoculation with an actively growing culture. Sterile controls were conducted alongside enrichment culture experiments. All conditions were tested in duplicate. Experiments were conducted in crimp-sealed vials and were incubated in the dark at $30{ }^{\circ} \mathrm{C}$ with gentle agitation for 4 days. DNA from enrichment cultures was extracted using Qiagen Blood and Tissue kits, following the manufacturer's instructions. Biomass was quantified using $\mathrm{OD}_{600}$ or, in cases where precipitates were formed, the BCA total protein assay (Thermo Scientific).

Microbial Community Analysis. The degenerate arsM primers arsM-309F (5' - GYI WWN GGI VTN GAY ATG A$\left.3^{\prime}\right)$ and arsM-470R (5'- ARR TTI AYI ACR CAR TTN S-3') were developed to amplify a greater diversity of ars $M$ sequences than are targeted with existing PCR primers. ${ }^{9}$ The I in the nucleotide sequence is inosine. Primer design was based on two amino acid motifs (motif-1: $[\mathrm{V} / \mathrm{A}][\mathrm{I} / \mathrm{Y}] \mathrm{G}[\mathrm{V} / \mathrm{I} / \mathrm{L}] \mathrm{DM}[\mathrm{T} / \mathrm{N}]$; motif-2: SNCV $[\mathrm{I} / \mathrm{V}] \mathrm{NL}$ ) found in a manually curated database of 212 sequences obtained by BLASTP using the Desulfovibrio desulfuricans ArsM protein sequence as a query. $D$. desulfuricans was chosen because it is an SRB and its arsM sequence is abundant in microarray surveys of diverse soils. ${ }^{8,11}$ The targeted amino acid motif- 2 covers one of the three conserved cysteines which are essential residues for the As methylating activity of ArsM enzymes. ${ }^{23,24}$ Details of the PCR amplification are available in SI. arsM amplicon sequencing was performed at the Research and Testing Laboratory in Lubbock, Texas, using paired-end Illumina MiSeq. A custom ArsM protein sequence database was built by searching for "ArsM" in the NCBI RefSeq database, filtering for protein lengths between 200 and 400, removing outliers, and aligning with Clustal Omega. The resulting database contained 726 sequences, a significant improvement in coverage over existing ars $M$ databases. ${ }^{21}$

The evolutionary placement of arsM (clustered at $90 \%$ similarity) on an arsM reference tree is described in detail in SI. In order to compare the ars $M$ diversity assessed through this study to previous investigations, ars $M$ gene sequences amplified by arsMF1/arsMR2 primers designed by Jia et al. and published in previous studies $9,18,22$ were retrieved from NCBI, translated to protein sequences, and clustered into OTUs.

$16 \mathrm{~S}$ rRNA gene diversity was assessed through paired-end Illumina MiSeq sequencing of the V4 region of the 16S rRNA gene. $^{38}$ Details are available in SI. arsM gene sequences determined in this study have been deposited in the European Nucleotide Archive with accession numbers LT860219LT882482 and $16 \mathrm{~S}$ gene sequences have been deposited in the NCBI GenBank database with accession numbers MF547744 - MF554589.

Chemical Analyses. Samples for aqueous As speciation were passed through a $0.22 \mu \mathrm{m}$ filter and stored in $0.24 \mathrm{M} \mathrm{HCl}$ at $-20{ }^{\circ} \mathrm{C}$ to preserve As species. ${ }^{8}$ To quantify mineral or cell surface-bound and intracellular As, unfiltered samples were periodically collected and preserved in $2 \mathrm{M} \mathrm{HCl}$ to dissolve $\mathrm{FeS}$ or other mineral phases. These samples were then extracted with either (a) $10 \mathrm{mM}$ EDTA to disrupt Gram-negative cell membranes or (b) a cell lysis buffer containing $0.1 \%$ Triton $\mathrm{X} 100,0.1 \%$ SDS, $10 \mathrm{mM}$ EDTA, and $1 \mathrm{mM}$ Tris- $\mathrm{HCl}$ to release intracellular As. Volatile As species were measured by purging the serum vial headspace at the end of the 4 day incubation and trapping volatile species on a chemo-trap consisting of a silica gel tube impregnated with $1 \% \mathrm{AgNO}_{3} .{ }^{39}$ The gel was then extracted with $1 \% \mathrm{HNO}_{3}$ at $90{ }^{\circ} \mathrm{C}$ for $1 \mathrm{~h}$, which converts trapped arsines to nonvolatile pentavalent oxyanion derivatives. Extracts were filtered through a $0.22 \mu \mathrm{M}$ filter, and stored at $-20{ }^{\circ} \mathrm{C}$ until analysis via high performance liquid chromatography-inductively coupled mass spectrometry (PerkinElmer Series 200 HPLC hyphenated to a PerkinElmer Elan ICP-MS), using a PRP X-100 anion exchange column. The eluent was $6.66 \mathrm{mM} \mathrm{NH}_{4} \mathrm{NO}_{3}$ and $6.66 \mathrm{mM} \mathrm{NH}_{2} \mathrm{H}_{2} \mathrm{PO}_{4}$, adjusted to $\mathrm{pH}$ 6.2 using $\mathrm{HNO}_{3}$. The ICP-MS was operated in oxygen reaction mode, so that As was measured at $m / z=91$ in order to avoid interference with $\mathrm{ArCl}$ at $m / z=75$. Prior to As analysis, $100 \mu \mathrm{L}$ hydrogen peroxide $\left(\mathrm{H}_{2} \mathrm{O}_{2}\right)$ was added to $400 \mu \mathrm{L}$ samples and stored overnight at $4{ }^{\circ} \mathrm{C}$ to oxidize $\mathrm{As}(\mathrm{III})$ to $\mathrm{As}(\mathrm{V})$ in order to reveal the presence of $\mathrm{TMAsO}(\mathrm{V})$ or other cationic or neutral As species that coelute with $\mathrm{As}(\mathrm{III})$. The $\mathrm{H}_{2} \mathrm{O}_{2}$ addition quantitatively converted As(III) to As (V) and did not affect the abundance of MMAs(V) or DMAs(V) (SI Figure S2). $\mathrm{SO}_{4}{ }^{2-}$ and $\mathrm{NO}_{3}{ }^{-}$were determined using ion chromatography (Dionex ICS-5000) and $\mathrm{Fe}(\mathrm{II})$ was determined using the ferrozine assay. $^{40}$

\section{RESULTS}

Biogeochemical Evolution of Enrichment Cultures. Enrichment culture EA exhibited $\mathrm{NO}_{3}{ }^{-}$-, $\mathrm{Fe}(\mathrm{III})$-, and $\mathrm{SO}_{4}{ }^{2-}$ reducing metabolisms. $\mathrm{NO}_{3}{ }^{-}$and $\mathrm{Fe}(\mathrm{III})$ reduction occurred simultaneously within the first $24 \mathrm{~h}$, followed by $\mathrm{SO}_{4}{ }^{2-}$ reduction between 20 and $60 \mathrm{~h}$ (Figure 1A). Both 5 and 20 $\mathrm{mM}$ molybdate, as well as $10 \mathrm{mM}$ monofluorophosphate, were effective at inhibiting $\mathrm{SO}_{4}{ }^{2-}$ reduction, while $20 \mathrm{mM}$ molybdate also slightly impacted $\mathrm{NO}_{3}^{-}$reduction (Figure $1 \mathrm{~A}$ and SI Figure S8). Total biomass levels in inhibited and uninhibited cultures, measured as total protein, were similar initially, but after $20 \mathrm{~h}$ the molybdate- and monofluorophosphate-inhibited cultures exhibited lower biomass levels (SI Figure S7 and S8B). In the presence of $\mathrm{SO}_{4}{ }^{2-}$ reduction, $\mathrm{Fe}(\mathrm{II})$ was titrated out of solution by sulfide (Figure 1A) and dark precipitates, likely FeS, were formed (SI Figure S1).

Arsenic Methylation Efficiency. Enrichment EA efficiently methylated As(III), converting $63 \%$ of the As(III) into either DMAs(V) or MMAs(V), with DMAs(V) as the dominant product (Figure 1B). Arsenic methylation was significantly reduced in the molybdate-inhibited experiments, where only $12 \%$ of the iAs was methylated. Essentially all of the iAs was removed from solution in enrichment EA, while in the molybdate-inhibited experiment, approximately $50 \%$ of the iAs remained in solution. Figure 2 summarizes methylation efficiencies in the different experimental variations of culture media EA after 100 h. Methylation efficiency (ME) for each 

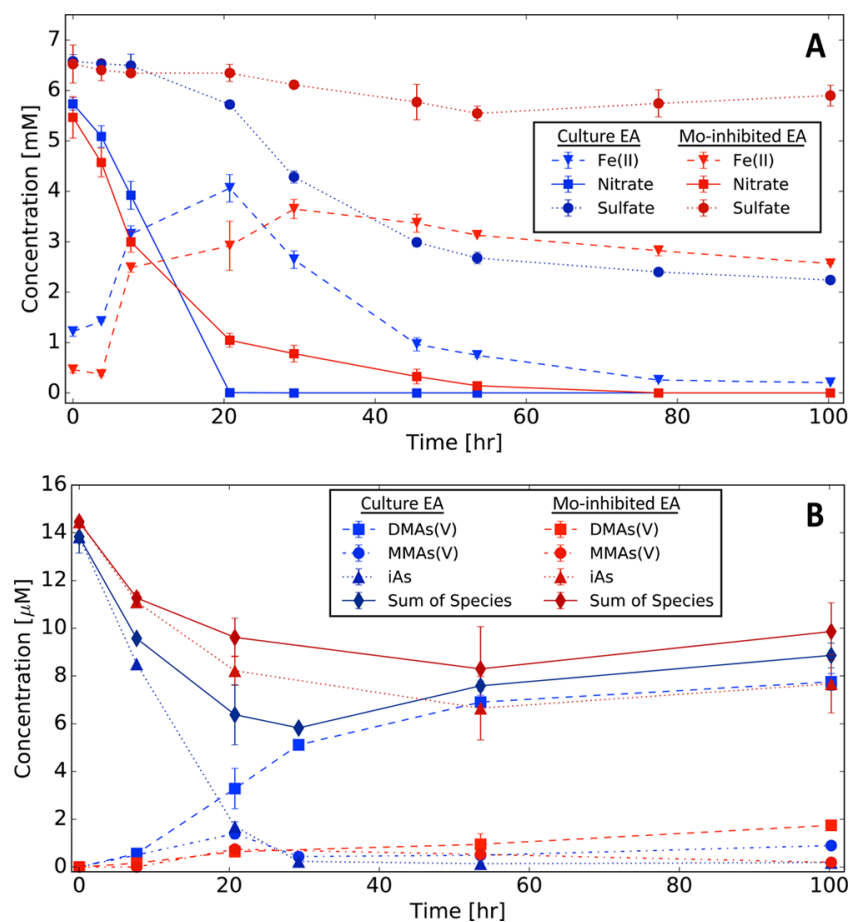

Figure 1. Evolution of enrichment culture chemistry. (A) Electron acceptor concentrations in enrichment culture EA and in the $20 \mathrm{mM}$ molybdate-inhibited enrichment culture EA. Symbols represent means and error bars show the range of duplicate vials; (B) Arsenic speciation in enrichment culture EA and in the $20 \mathrm{mM}$ molybdate-inhibited enrichment culture EA. Symbols represent means and error bars show the range of duplicate vials. In some cases error bars are smaller than the symbols.

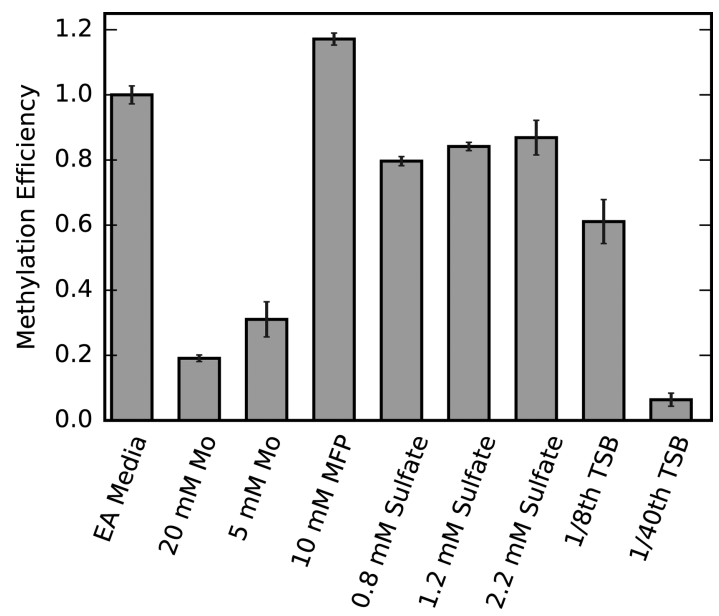

Figure 2. Methylation efficiency of enrichment culture EA and in modified EA enrichment cultures, normalized to the efficiency observed for unmodified EA conditions. MFP is monofluorophosphate and Mo is molybdate. Data on $\mathrm{SO}_{4}{ }^{2-}$ reduction in monofluorophosphate-inhibited experiments and experiments with low $\mathrm{SO}_{4}{ }^{2-}$ concentrations are shown in SI Figure S8.

experimental condition $i$ is expressed relative to the methylation efficiency of a parallel experiment performed with the standard EA media to account for differences between batch experiments inoculated with different glycerol stocks:

$$
\operatorname{ME}_{i}=\frac{\operatorname{MMAs}(\mathrm{V})_{i}+\operatorname{DMAs}(\mathrm{V})_{i}}{\operatorname{MMAs}(\mathrm{V})_{\mathrm{EA}}+\operatorname{DMAs}(\mathrm{V})_{\mathrm{EA}}}
$$

Volatile As species, as well as cell-associated $\operatorname{MMAs}(\mathrm{V})$ and $\operatorname{DMAs}(\mathrm{V})$, were not included in the calculation since they were not measured in every experiment. When measured, cellassociated MMAs(V) and DMAs(V) concentrations were very small relative to aqueous MMAs(V) and DMAs(V) (SI Figure S10).

Unlike molybdate, the monofluorophosphate-inhibited media did not exhibit a reduction in methylation efficiency while it did effectively inhibit $\mathrm{SO}_{4}{ }^{2-}$ reduction (Figure 2 and SI Figure S8A). The methylation efficiency was also insensitive to the initial $\mathrm{SO}_{4}{ }^{2-}$ concentration, with media containing $0.8,1.2$, 2.2 , and $6.1 \mathrm{mM} \mathrm{SO}_{4}{ }^{2-}$ yielding similar methylation efficiencies (Figure 2). $\mathrm{SO}_{4}{ }^{2-}$ was completely reduced in each of these cases (SI Figure S8A). Methylation efficiency was effectively limited by reducing the concentration of TSB in the EA medium, with the reduction of TSB from $1 / 4$ to $1 / 40$ strength causing a $>90 \%$ reduction in methylation efficiency. Reducing the TSB concentration reduced the biomass concentrations and the extent of $\mathrm{SO}_{4}{ }^{2-}$ reduction, with approximately $1 / 3 \mathrm{rd}$ of the $\mathrm{SO}_{4}{ }^{2-}$ remaining after $100 \mathrm{~h}$, while in the standard EA media $\mathrm{SO}_{4}{ }^{2-}$ was completely reduced (SI Figure S8A). iAs, MMAs$(\mathrm{V})$, and DMAs(V) were stable in sterile EA media (SI Figure S9).

When amended with MMAs(V), approximately $50 \%$ of the MMAs(V) was removed from solution and approximately $15 \%$ of the original MMAs $(\mathrm{V})$ was further methylated to DMAs $(\mathrm{V})$ (Figure 3). In contrast, aqueous DMAs(V) concentrations were

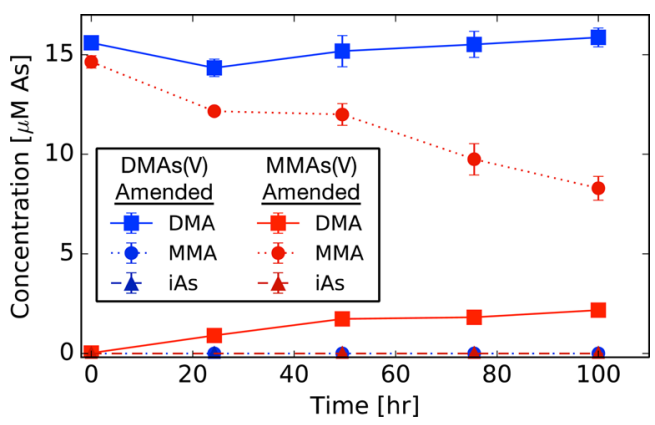

Figure 3. Evolution of arsenic species in enrichment culture EA when amended with MMAs(V) or DMAs(V). Symbols represent means and error bars represent the range of duplicate vials.

conservative over $100 \mathrm{~h}$, indicating that further methylation to trimethylated species or demethylation was negligible. No iAs was detected in the culture media in either the DMAs $(V)$ - or $\operatorname{MMAs}(\mathrm{V})$-amended vials, indicating the lack of a demethylating capability in the culture.

Arsenic Mass Balances. Aqueous mass balances of As revealed significant mass of As that was unaccounted for (Figure 1B). The extraction of unfiltered culture samples with 2 $\mathrm{M} \mathrm{HCl}$, followed by extraction with either EDTA or cell lysis buffer, showed that EDTA and the cell lysis buffer solubilized substantial iAs relative to the $2 \mathrm{M} \mathrm{HCl}$ solution (SI Figure $\mathrm{S} 10)$. Aqueous iAs concentrations increased from $1.6 \mu \mathrm{M}$ in the $2 \mathrm{M} \mathrm{HCl}$ solution to $9.3 \mu \mathrm{M}$ in the lysis buffer solution, indicating that a significant fraction of the iAs was bound to cell surfaces or had been taken up into cells. MMAs(V) and DMAs $(V)$ concentrations did not vary significantly between the different extractions. The sample treated with the lysis buffer showed a complete mass recovery of As.

DMAs $(V)$ was detected in the chemo-trap extract from the molybdate-inhibited EA enrichment experiment (SI Figure 


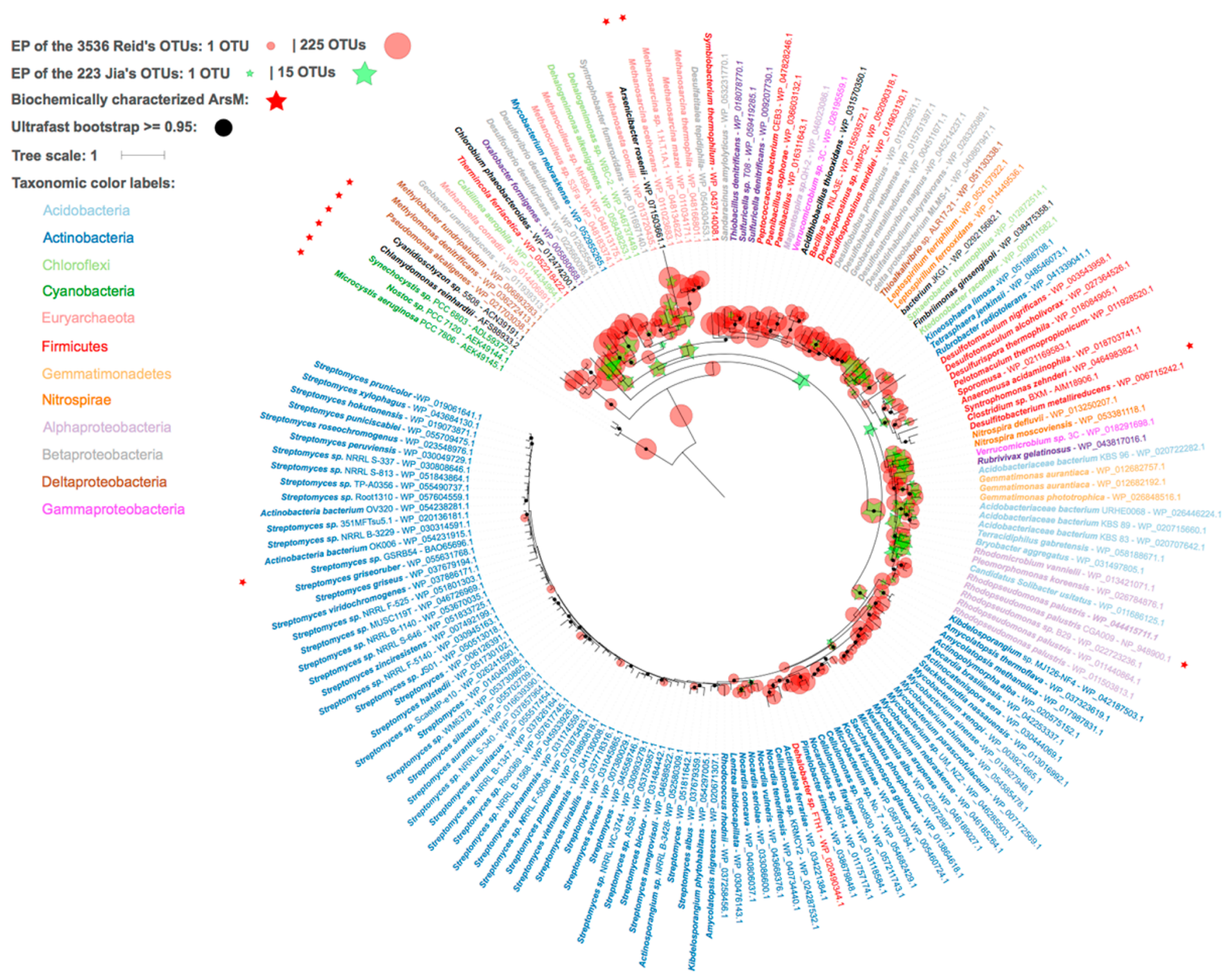

Figure 4. Evolutionary placement of ArsM OTUs (90\% OTUs) on a rooted maximum likelihood tree composed of 175 reference proteins sequences (90\% OTUs), generated with IQ-TREE, based on a LG+F+I+G4 substitution model. ${ }^{51,52}$ Each OTU placement is indicated by a red circle (for OTUs sequenced using the new primers set from the paddy soil sample) or by a green star (for OTUs sequenced using arsMF1/arsMR2 primers set), the size of these symbols representing the number of OTUs falling in each of these placements. Biochemically characterized ArsM proteins present on the reference tree are indicated with a red star. Ultrafast bootstrap values greater than $95 \%$ are indicated with a black circle. Tree leaves are color-coded according to the phylum (or class for Proteobacteria) classification of the reference organisms. See the tree legend for the color coding.

S11), accounting for $3.3 \mathrm{nmol}$ of volatilized As (or $0.6 \%$ of the total As). Additional discussion of the volatile As species is in SI.

Microbial Diversity in Paddy Soil and Enrichment Cultures. Figure 4 shows the evolutionary placements of ars $M$ OTUs (clustered at 90\%; red dots) amplified with arsM-390F/ arsM-470R primers from the original paddy soil on a reference tree based on the 726 ArsM protein database. The diversity covered by this new primer set is significantly broader than the one covered by arsMF1/arsMR2, as evidenced by the sparse placement of OTUs (also clustered at 90\%; green stars) originating from 16 different samples amplified with the latter primer set. $9,18,22$ The new primers allow the amplification of Actinobacteria, Firmicutes, Cyanobacteria, Euryarchaeota, Beta-, Delta-, and Gamma-proteobacteria arsM genes. SI Figure S4 shows the result of the analysis based on a random selection of 531 reads to account for the greater sequencing depth of Illumina MiSeq technology used here versus the cloning- sequencing used with arsMF1/arsMR2 primers. While the density of the placements for arsM-390F/arsM-470R OTUs (90\%) is lower than in Figure 4, the diversity range is still broader than that of arsMF1/arsMR2 OTUs.

Phylogenetic diversity of microbial 16S rRNA genes in the paddy soil, in enrichment EA, and in the molybdate-inhibited EA culture is shown in SI Figure S5. In the paddy soil, the classes Acidobacteria and Alpha-, Beta-, Gamma-, and Deltaproteobacteria contributed approximately $50 \%$ of the diversity, while $33 \%$ was not classifiable at the class level. Actinobacter, Clostridia, Bacteroidia, and Bacilli also contributed to the prokaryotic diversity. Over 3500 arsM OTUs were identified in the paddy soil, and were distributed across diverse microbial phyla (Figure 4). Less than $10 \%$ of the arsM OTUs identified in the paddy soil could be taxonomically classified at the class level, preventing a comparative analysis of $16 \mathrm{~S}$ rRNA and $\operatorname{ars} M$ gene diversity. 
There was a shift in the microbial community structure between the paddy soil and the enrichment cultures used in methylation experiments. The classes Clostridia, Bacilli, Bacteroidia, and Deltaproteobacteria represented a larger fraction of the prokaryotic $16 \mathrm{~S}$ rRNA gene sequences in enrichment EA relative to the original soil, while the abundance of Alphaproteobacteria decreased (SI Figure S5). The class Negativicutes, which includes the Anaeromusa genus, increased to $>1 \%$ of the prokaryotic abundance in the EA enrichment. Molybdate inhibition caused a reduction in the relative abundance of Deltaproteobacteria from $34 \%$ to $<1 \%$, with an increase in the relative abundance of Gammaproteobacteria and Clostridia. Diversity of the ars $M$ sequences in the enrichments was low, with $83 \%$ of the reads contributed by 3 OTUs in enrichment EA and $75 \%$ of the reads contributed by 4 OTUs in the molybdate-inhibited culture. OTUs identified in the enrichments were members of the Proteobacteria and Firmicutes, with Proteobacteria representing a larger fraction in EA relative to the molybdate-inhibited culture (SI Figure S6). $32 \%$ of the reads in EA could be classified at the genus level, with the genera Anaeromusa and Desulfovibrio representing 24\% and 8\%, respectively. The genus Anaeromusa consists only of the species Anaeromusa acidaminophila, which is an amino acid fermenting bacterium. ${ }^{41}$ In the molybdate-inhibited culture the abundance of Anaeromusa was unchanged, whereas Desulfovibrio decreased to $4 \%$.

\section{DISCUSSION}

Microbial Metabolism and Arsenic Methylation Efficiency. The enrichment cultures efficiently transformed As(III) to methylated products, with more than $63 \%$ of the As(III) converted to either MMAs(V) or DMAs(V) over the $100 \mathrm{~h}$ incubation. DMAs(V) was the major product. Previous assays of As methylation by both pure and mixed anaerobic cultures showed less efficient methylation than that observed here (Table 1), with $<15 \%$ of the initial iAs transformed to methylated species. Results of the present study also differed from some previous anaerobic studies in that $\operatorname{DMAs}(\mathrm{V})$ and not MMAs(V) was the dominant methylated product. ${ }^{23,24} \mathrm{An}$ explanation for prior observations of greater methylation efficiency in aerobic relative to anaerobic cultures is that toxic trivalent methylated species are quickly oxidized to less toxic pentavalent derivatives in aerobic systems, ${ }^{15}$ precluding the accumulation of the toxic form of As. The anaerobic culture examined in this study diverged significantly from the pattern of limited methylation by anaerobic cultures, however, demonstrating that robust arsenic methylation is possible in anaerobic systems.

The reduction of arsenic methylation efficiency in the presence of molybdate could initially be interpreted to suggest that SRB are principal drivers of arsenic methylation (Figure 1), but further confirmatory tests indicated that the effect of molybdate was unrelated to its role in inhibiting $\mathrm{SO}_{4}{ }^{2-}$ reduction and instead involved an inhibition of the methylation pathway. In particular, observations of robust methylation independent of $\mathrm{SO}_{4}{ }^{2-}$ concentrations relevant to dissimilatory sulfate reduction (DSR) (Figure 2) as well as robust methylation despite clear inhibition of $\mathrm{SO}_{4}{ }^{2-}$ reduction by monofluorophosphate (SI Figure S8A) indicate that SRB are not principal contributors to arsenic methylation in these enrichment cultures. The mechanism for molybdate and monofluorophosphate inhibition of sulfate reduction is the same: reaction between molybdate or monofluorophosphate and ATP interferes with the first step in the DSR pathway, the formation of adenosine 5 '-phosphosulfate (APS) from ATP and $\mathrm{SO}_{4}{ }^{2-}$. The fact that addition of recommended concentrations $^{36}$ of molybdate versus monofluorophosphate had divergent effects on As methylation suggests that the mechanism was not solely related to the inhibition of DSR. Higher molybdate concentrations caused greater reductions in methylation efficiency (Figure 2), suggesting a dose-dependent effect of molybdate on arsenic methylation. The specific mechanism of this inhibition remains unclear.

While nearly all of the aqueous As was transformed to methylated species in enrichment EA, a significant fraction of As in the system was removed from the aqueous phase and persisted as biomass-associated iAs (SI Figure S10). Extractions of acidified, unfiltered culture samples with EDTA or a lysis buffer suggest that the iAs was sequestered partly via cellular uptake and accumulation. MMAs(V) and DMAs(V) were unaffected by these removal processes in experiments amended with As(III), though in the culture amended with MMAs(V) only a fraction of the MMAs(V) taken up from solution was released as DMAs(V) (Figure 3). Recently, Huang et al. ${ }^{29}$ argued that the efficient arsenic methylation observed in $A$. rosenii is due partly to the lack of As(III) efflux transporters, leading to intracellular accumulation of As(III) that favors methylation reactions. As(III) uptake via aquaglyceroporins is widespread in prokaryotes ${ }^{42}$ so it is not possible to discern which microorganisms in the enrichments could accumulate As(III).

One reason for the efficient production of methylated species in the enrichments was that the culture lacked the ability to demethylate MMAs(V) or DMAs(V), though methylation of MMAs(V) to DMAs(V) was observed (Figure 3). Rapid demethylation of methylated As has been proposed as an explanation for why environmental abundances of methylated arsenic remain low. ${ }^{43}$ Arsenic demethylation pathways by aerobes have been characterized ${ }^{44,45}$ and a C-As lyase has been identified. ${ }^{43}$ Studies of As demethylation under anaerobic conditions have yielded conflicting results, however. SierraAlvarez et al. $^{46}$ observed demethylation of MMAs(V) and DMAs(V) under sulfate-reducing and methanogenic conditions, while results in Huang et al. ${ }^{47}$ were similar to those in this study and showed that DMAs(V) was stable in an anoxic soil incubation. Further research into the role of demethylation in controlling methylated arsenic balances in anaerobic environments is warranted.

Microbial Community Structure and Biomethylation Function. Enrichment cultures were characterized by a low level of microbial diversity relative to the original paddy soil but maintained representatives of several classes (SI Figure S5), including multiple microorganisms harboring arsM genes (SI Figure S6). arsM sequences from the genera Anaeromusa and Desulfovibrio dominated the enrichment culture. The diversity in the molybdate-inhibited culture shifted to have a smaller fraction of Deltaproteobacteria, likely due to inhibited growth of Desulfovibrio spp. The presence of Desulfovibrio harboring arsM in the enrichments provided an opportunity to examine the role of an SRB in As methylation.

As discussed in the previous section, arsenic biomethylation was decoupled from DSR activity. Some Desulfovibrio species can grow on nitrate, ${ }^{48}$ which could explain why Desulfovibrio $\operatorname{ars} M$ is present, albeit at a lower relative abundance, in the molybdate-inhibited enrichment. The greatest reductions in As methylation efficiency were observed when TSB concentra- 
tions, and thus the fermentable amino acids, were decreased (Figure 2). While these results do not allow for a definitive conclusion of the microorganisms driving As biomethylation in the enrichments, they suggest that the amino acid-fermenting organisms (potentially A. acidaminophila) may play a role in the observed methylation. The possibility also remains that methylating microorganisms were not amplified by the new primer pairs, or that amplified ars $M$ fragments could not be identified due to limitations of the database.

Implications for Methylated Arsenic in Paddy Environments. Determining the key microorganisms involved in paddy soil arsenic biomethylation, and consequently the biogeochemical controls on methylation efficiencies, has been identified as a priority for understanding As speciation in rice. ${ }^{10}$ This study supports previous investigations showing that paddy soil microorganisms harboring arsM are functionally and phylogenetically diverse, while also revealing a greater depth of ars $M$ diversity than has been shown previously. The ars $M$ diversity range detected by the highly degenerate ars $M$ primer set, arsM-309F/arsM-470R, in a single paddy sample is broader than that detected in samples from diverse environments amplified by arsMF1/arsMR2 primers. While this analysis can only be qualitative due to the different sequencing technologies employed, the analysis performed by randomly subsampling 531 reads from arsM-309F/arsM-470R amplicons (SI Figure S4) further demonstrates the broader diversity range surveyed with these new primers. Assessments of As methylation in soils and other environments have been hindered partly by limited databases for taxonomic classification..$^{20,21,49}$ The more extensive 726 protein sequence database developed for this study, along with a primer pair having greater degeneracy to amplify a broad range of ars $M$ sequences, has enabled a deeper examination of arsM phylogenetic diversity. This study demonstrates the utility of these new tools, in conjunction with analyses of physiological responses to As, in probing relationships between community structure and biomethylation function.

This study focused on the role of SRB in As biomethylation through the development of metabolically versatile mixed anaerobic enrichments from a rice paddy soil and a series of molybdate- and monofluorophosphate-inhibited experiments to evaluate the role of SRB. An arsM gene diversity analysis and the physiological response of the enrichment culture suggest that methylation was decoupled from DSR activity, and that amino acid fermenting microorganisms may play a role in the observed methylation. Because the diversity of the enrichment cultures differed from that of the paddy soil, the conclusions on the ecological roles of specific microorganisms are not generalizable to natural environments. The anaerobic enrichment culture developed in this study exhibited a methylation capacity that was significantly greater than that observed in previous studies with anaerobes. While the reasons are not clear, the lack of a demethylating capacity underscores the need for further investigations into arsenic demethylation in order to understand the controls on methylated arsenic species in the environment.

\section{ASSOCIATED CONTENT}

\section{S Supporting Information}

The Supporting Information is available free of charge on the ACS Publications website at DOI: 10.1021/acs.est.7b02970.

Additional data and all experimental details (PDF)

\section{AUTHOR INFORMATION}

\section{Corresponding Author}

*Phone: 607-255-6082; fax: 607-255-9004; e-mail: mcr239@ cornell.edu.

ORCID

Matthew C. Reid: 0000-0001-5185-7678

\section{Present Addresses}

"School of Civil and Environmental Engineering, Cornell University, Ithaca, New York 14853, United States.

${ }^{\perp}$ Department of Ecogenomics and Systems Biology, University of Vienna, Vienna, Austria.

\section{Notes}

The authors declare no competing financial interest.

\section{ACKNOWLEDGMENTS}

We thank M. Frutschi and V. Pham for assistance with soil sampling in Vietnam. This project received funding from the EPFL Fellows' fellowship program cofunded by Marie Skłodowska Curie (European Union's Seventh Framework Program for research, technological development and demonstration) under grant agreement no 291771. Additional funding was provided by the EPFL Integrative Food Science and Nutrition Center. The complete scripts of bioinformatics analyses can be found at https://github.com/alex-bagnoud/ ArsM-evolutionary-placements

\section{REFERENCES}

(1) Williams, P.; Price, A.; Raab, A.; Hossain, S.; Feldmann, J.; Meharg, A. Variation in arsenic speciation and concentration in paddy rice related to dietary exposure. Environ. Sci. Technol. 2005, 39 (15), $5531-5540$.

(2) Zavala, Y. J.; Gerads, R.; Gürleyük, H.; Duxbury, J. M. Arsenic in rice: II. Arsenic speciation in USA grain and implications for human health. Environ. Sci. Technol. 2008, 42 (10), 3861-3866.

(3) Zhao, F.-J.; McGrath, S. P.; Meharg, A. A. Arsenic as a food chain contaminant: mechanisms of plant uptake and metabolism and mitigation strategies. Annu. Rev. Plant Biol. 2010, 61, 535-559.

(4) Somenahally, A. C.; Hollister, E. B.; Yan, W.; Gentry, T. J.; Loeppert, R. H. Water management impacts on arsenic speciation and iron-reducing bacteria in contrasting rice-rhizosphere compartments. Environ. Sci. Technol. 2011, 45 (19), 8328-8335.

(5) Roberts, L. C.; Hug, S. J.; Voegelin, A.; Dittmar, J.; Kretzschmar, R.; Wehrli, B.; Saha, G. C.; Badruzzaman, A. B. M.; Ali, M. A. Arsenic dynamics in porewater of an intermittently irrigated paddy field in Bangladesh. Environ. Sci. Technol. 2010, 45 (3), 971-976.

(6) Dittmar, J.; Voegelin, A.; Maurer, F.; Roberts, L. C.; Hug, S. J.; Saha, G. C.; Ali, M. A.; Badruzzaman, A. B. M.; Kretzschmar, R. Arsenic in soil and irrigation water affects arsenic uptake by rice: complementary insights from field and pot studies. Environ. Sci. Technol. 2010, 44 (23), 8842-8848.

(7) Williams, P. N.; Zhang, H.; Davison, W.; Meharg, A. A.; Hossain, M.; Norton, G. J.; Brammer, H.; Islam, M. R. Organic Matter-Solid Phase Interactions Are Critical for Predicting Arsenic Release and Plant Uptake in Bangladesh Paddy Soils. Environ. Sci. Technol. 2011, 45 (14), 6080-6087.

(8) Zhao, F.-J.; Harris, E.; Yan, J.; Ma, J.; Wu, L.; Liu, W.; McGrath, S. P.; Zhou, J.; Zhu, Y.-G. Arsenic methylation in soils and its relationship with microbial ars $M$ abundance and diversity, and As speciation in rice. Environ. Sci. Technol. 2013, 47 (13), 7147-7154.

(9) Jia, Y.; Huang, H.; Zhong, M.; Wang, F.-H.; Zhang, L.-M.; Zhu, Y.-G. Microbial arsenic methylation in soil and rice rhizosphere. Environ. Sci. Technol. 2013, 47 (7), 3141-3148.

(10) Zhao, F.-J.; Zhu, Y.-G.; Meharg, A. A. Methylated arsenic species in rice: geographical variation, origin, and uptake mechanisms. Environ. Sci. Technol. 2013, 47 (9), 3957-3966. 
(11) Lomax, C.; Liu, W. J.; Wu, L.; Xue, K.; Xiong, J.; Zhou, J.; McGrath, S. P.; Meharg, A. A.; Miller, A. J.; Zhao, F. J. Methylated arsenic species in plants originate from soil microorganisms. New Phytol. 2012, 193 (3), 665-672.

(12) Zhu, Y.-G.; Yoshinaga, M.; Zhao, F.-J.; Rosen, B. P. Earth abides arsenic biotransformations. Annu. Rev. Earth Planet. Sci. 2014, 42, 443.

(13) Meharg, A. A.; Lombi, E.; Williams, P. N.; Scheckel, K. G.; Feldmann, J.; Raab, A.; Zhu, Y.; Islam, R. Speciation and localization of arsenic in white and brown rice grains. Environ. Sci. Technol. 2008, 42 (4), 1051-1057.

(14) Qin, J.; Rosen, B. P.; Zhang, Y.; Wang, G.; Franke, S.; Rensing, C. Arsenic detoxification and evolution of trimethylarsine gas by a microbial arsenite S-adenosylmethionine methyltransferase. Proc. Natl. Acad. Sci. U. S. A. 2006, 103 (7), 2075-2080.

(15) Li, J.; Pawitwar, S. S.; Rosen, B. P. The organoarsenical biocycle and the primordial antibiotic methylarsenite. Metallomics 2016, 8 (10), 1047-1055.

(16) Bentley, R.; Chasteen, T. G. Microbial methylation of metalloids: arsenic, antimony, and bismuth. Microbiology and Molecular Biology Reviews 2002, 66 (2), 250-271.

(17) Zhu, Y.-G.; Xue, X.-M.; Kappler, A.; Rosen, B. P.; Meharg, A. A. Linking genes to microbial biogeochemical cycling: lessons from arsenic. Environ. Sci. Technol. 2017, 51, 7326.

(18) Zhang, S.-Y.; Zhao, F.-J.; Sun, G.-X.; Su, J.-Q.; Yang, X.-R.; Li, H.; Zhu, Y.-G. Diversity and abundance of arsenic biotransformation genes in paddy soils from southern China. Environ. Sci. Technol. 2015, 49 (7), 4138-4146.

(19) Gu, Y.; Van Nostrand, J. D.; Wu, L.; He, Z.; Qin, Y.; Zhao, F.-J.; Zhou, J. Bacterial community and arsenic functional genes diversity in arsenic contaminated soils from different geographic locations. PLoS One 2017, 12 (5), e0176696.

(20) Zhai, W.; Wong, M. T.; Luo, F.; Hashmi, M. Z.; Liu, X.; Edwards, E. A.; Tang, X.; Xu, J. Arsenic Methylation and its Relationship to Abundance and Diversity of arsM Genes in Composting Manure. Sci. Rep. 2017, 7, 7.

(21) Cai, L.; Yu, K.; Yang, Y.; Chen, B.-w.; Li, X.-d.; Zhang, T. Metagenomic exploration reveals high levels of microbial arsenic metabolism genes in activated sludge and coastal sediments. Appl. Microbiol. Biotechnol. 2013, 97 (21), 9579-9588.

(22) Desoeuvre, A.; Casiot, C.; Héry, M. Diversity and Distribution of Arsenic-Related Genes Along a Pollution Gradient in a River Affected by Acid Mine Drainage. Microb. Ecol. 2016, 71 (3), 672-685.

(23) Wang, P.-P.; Sun, G.-X.; Zhu, Y.-G. Identification and characterization of arsenite methyltransferase from an archaeon, Methanosarcina acetivorans C2A. Environ. Sci. Technol. 2014, 48 (21), 12706-12713.

(24) Wang, P.-P.; Bao, P.; Sun, G.-X. Identification and catalytic residues of the arsenite methyltransferase from a sulfate-reducing bacterium, Clostridium sp. BXM. FEMS Microbiology Letters 2015, 362 (1), $1-8$.

(25) Kuramata, M.; Sakakibara, F.; Kataoka, R.; Abe, T.; Asano, M.; Baba, K.; Takagi, K.; Ishikawa, S. Arsenic biotransformation by Streptomyces sp. isolated from rice rhizosphere. Environ. Microbiol. 2015, 17 (6), 1897-1909.

(26) Zhang, J.; Cao, T.; Tang, Z.; Shen, Q.; Rosen, B. P.; Zhao, F.-J. Arsenic methylation and volatilization by arsenite $S$-adenosylmethionine methyltransferase in Pseudomonas alcaligenes NBRC14159. Appl. Environ. Microbiol. 2015, 81 (8), 2852-2860.

(27) Huang, K.; Chen, C.; Zhang, J.; Tang, Z.; Shen, Q.; Rosen, B. P.; Zhao, F.-J. Efficient Arsenic Methylation and Volatilization Mediated by a Novel Bacterium From an Arsenic-Contaminated Paddy Soil. Environ. Sci. Technol. 2016, 50, 6389-6396.

(28) Mestrot, A.; Feldmann, J.; Krupp, E. M.; Hossain, M. S.; Roman-Ross, G.; Meharg, A. A. Field fluxes and speciation of arsines emanating from soils. Environ. Sci. Technol. 2011, 45 (5), 1798-1804.

(29) Huang, H.; Jia, Y.; Sun, G.-X.; Zhu, Y.-G. Arsenic speciation and volatilization from flooded paddy soils amended with different organic matters. Environ. Sci. Technol. 2012, 46 (4), 2163-2168.
(30) Bright, D.; Brock, S.; Reimer, K.; Cullen, W.; Hewitt, G.; Jafaar, $\mathrm{J}$. Methylation of arsenic by anaerobic microbial consortia isolated from lake sediment. Appl. Organomet. Chem. 1994, 8 (4), 415-422.

(31) Mikutta, C.; Rothwell, J. J. Peat Bogs as Hotspots for Organoarsenical Formation and Persistence. Environ. Sci. Technol. 2016, 50 (8), 4314-4323.

(32) Zeng, X.; Jiang, Y.; Fan, X.; Chao, S.; Yang, Y.; Liu, J.; Zhu, M.; Cao, H. Effects of Sulfate Application on Inhibiting Accumulation and Alleviating Toxicity of Arsenic in Panax notoginseng Grown in ArsenicPolluted Soil. Water, Air, Soil Pollut. 2016, 227 (5), 1-11.

(33) Gilmour, C. C.; Henry, E. A.; Mitchell, R. Sulfate stimulation of mercury methylation in freshwater sediments. Environ. Sci. Technol. 1992, 26 (11), 2281-2287.

(34) Compeau, G.; Bartha, R. Sulfate-reducing bacteria: principal methylators of mercury in anoxic estuarine sediment. Appl. Environ. Microbiol. 1985, 50 (2), 498-502.

(35) Parks, J. M.; Johs, A.; Podar, M.; Bridou, R.; Hurt, R. A.; Smith, S. D.; Tomanicek, S. J.; Qian, Y.; Brown, S. D.; Brandt, C. C. The genetic basis for bacterial mercury methylation. Science 2013, 339 (6125), 1332-1335.

(36) Carlson, H. K.; Stoeva, M. K.; Justice, N. B.; Sczesnak, A.; Mullan, M. R.; Mosqueda, L. A.; Kuehl, J. V.; Deutschbauer, A. M.; Arkin, A. P.; Coates, J. D. Monofluorophosphate is a selective inhibitor of respiratory sulfate-reducing microorganisms. Environ. Sci. Technol. 2015, 49 (6), 3727-3736.

(37) Fleming, E. J.; Mack, E. E.; Green, P. G.; Nelson, D. C. Mercury methylation from unexpected sources: molybdate-inhibited freshwater sediments and an iron-reducing bacterium. Appl. Environ. Microbiol. 2006, 72 (1), 457-464.

(38) Caporaso, J. G.; Lauber, C. L.; Walters, W. A.; Berg-Lyons, D.; Lozupone, C. A.; Turnbaugh, P. J.; Fierer, N.; Knight, R. Global patterns of $16 \mathrm{~S}$ rRNA diversity at a depth of millions of sequences per sample. Proc. Natl. Acad. Sci. U. S. A. 2011, 108 (Supplement 1), $4516-4522$

(39) Mestrot, A.; Uroic, M. K.; Plantevin, T.; Islam, M. R.; Krupp, E. M.; Feldmann, J. r.; Meharg, A. A. Quantitative and qualitative trapping of arsines deployed to assess loss of volatile arsenic from paddy soil. Environ. Sci. Technol. 2009, 43 (21), 8270-8275.

(40) Stookey, L. L. Ferrozine-a new spectrophotometric reagent for iron. Anal. Chem. 1970, 42 (7), 779-781.

(41) Baena, S.; Fardeau, M.-L.; Woo, T.; Ollivier, B.; Labat, M.; Patel, B. K. Phylogenetic relationships of three amino-acid-utilizing anaerobes, Selenomonas acidaminovorans, Selenomonas acidaminophila, and Eubacterium acidaminophilum, as inferred from partial 16S rDNA nucleotide sequences and proposal of Thermanaerovibrio acidaminovorans gen. nov., comb. nov. and Anaeromusa acidaminophila gen. nov., comb. nov. Int. J. Syst. Bacteriol. 1999, 49 (3), 969-974.

(42) Rosen, B. P.; Tamás, M. J., Arsenic transport in prokaryotes and eukaryotic microbes. In MIPs and Their Role in the Exchange of Metalloids; Springer, 2010; pp 47-55.

(43) Yoshinaga, M.; Rosen, B. P. AC. As lyase for degradation of environmental organoarsenical herbicides and animal husbandry growth promoters. Proc. Natl. Acad. Sci. U. S. A. 2014, 111 (21), 7701-7706.

(44) Yan, Y.; Ye, J.; Xue, X.-M.; Zhu, Y.-G. Arsenic demethylation by a C. As lyase in cyanobacterium Nostoc sp. PCC 7120. Environ. Sci. Technol. 2015, 49 (24), 14350-14358.

(45) Yoshinaga, M.; Cai, Y.; Rosen, B. P. Demethylation of methylarsonic acid by a microbial community. Environ. Microbiol. 2011, 13 (5), 1205-1215.

(46) Sierra-Alvarez, R.; Yenal, U.; Field, J. A.; Kopplin, M.; Gandolfi, A. J.; Garbarino, J. R. Anaerobic biotransformation of organoarsenical pesticides monomethylarsonic acid and dimethylarsinic acid. J. Agric. Food Chem. 2006, 54 (11), 3959-3966.

(47) Huang, J.-H.; Scherr, F.; Matzner, E. Demethylation of dimethylarsinic acid and arsenobetaine in different organic soils. Water, Air, Soil Pollut. 2007, 182 (1-4), 31-41.

(48) Dalsgaard, T.; Bak, F. Nitrate reduction in a sulfate-reducing bacterium, Desulfovibrio desulfuricans, isolated from rice paddy soil: 
sulfide inhibition, kinetics, and regulation. Appl. Environ. Microbiol. 1994, 60 (1), 291-297.

(49) Xiao, K.-Q.; Li, L.-G.; Ma, L.-P.; Zhang, S.-Y.; Bao, P.; Zhang, T.; Zhu, Y.-G. Metagenomic analysis revealed highly diverse microbial arsenic metabolism genes in paddy soils with low-arsenic contents. Environ. Pollut. 2016, 211, 1-8.

(50) Chen, J.; Sun, G.-X.; Wang, X.-X.; Lorenzo, V. c. d.; Rosen, B. P.; Zhu, Y.-G. Volatilization of arsenic from polluted soil by Pseudomonas putida engineered for expression of the arsM arsenic (III) S-adenosine methyltransferase gene. Environ. Sci. Technol. 2014, 48 (17), 10337-10344.

(51) Nguyen, L.-T.; Schmidt, H. A.; von Haeseler, A.; Minh, B. Q. IQ-TREE: a fast and effective stochastic algorithm for estimating maximum-likelihood phylogenies. Mol. Biol. Evol. 2015, 32 (1), 268274.

(52) Stamatakis, A. RAxML version 8: a tool for phylogenetic analysis and post-analysis of. Bioinformatics 2014, 30 (9), 1312-1313. 